\title{
Unital algebras of Hom-associative type and surjective or injective twistings
}

\author{
Yaël FRÉGIER ${ }^{a}$, Aron GOHR $^{a}$, and Sergei SILVESTROV ${ }^{b}$ \\ ${ }^{a}$ Mathematics Research Unit, University of Luxembourg, 162A Avenue de la faiencerie, \\ L-1511 Luxembourg, Grand-Duchy of Luxembourg \\ ${ }^{b}$ Centre for Mathematical Sciences, Lund University, Box 118, SE-221 00 Lund, Sweden \\ E-mails:yael.fregier@uni.lu,aron.gohr@uni.lu,ssilvest@maths.lth.se
}

\begin{abstract}
In this paper, we introduce a common generalizing framework for alternative types of Hom-associative algebras. We show that the observation that unital Hom-associative algebras with surjective or injective twisting map are already associative has a generalization in this new framework. We also show by construction of a counterexample that another such generalization fails even in a very restricted particular case. Finally, we discuss an application of these observations by answering in the negative the question whether nonassociative algebras with unit such as the octonions may be twisted by the composition trick into Hom-associative algebras.
\end{abstract}

2000 MSC: 17A30, 16Y99, 17A01, 17A20, 17D25

\section{Introduction}

Hom-Lie-algebras were first studied in the context of deformation of Witt and Virasoro algebras by Hartwig, Larsson, and Silvestrov in [7]. The basic idea underlying Hom-algebras, which amounts to generalization of a given type of algebraic structure by adjoining as an additional piece of data linear maps twisting the defining identities, has since been applied in many categories of algebraic objects. In $[7,8,12]$, quasi-Hom-Lie algebras, the general quasi-Lie algebras, and the corresponding generalization of Leibniz algebras quasi-Leibniz algebras [9] have been introduced. The graded versions, $\Gamma$-graded quasi-Lie algebras, were introduced in [10]. They contain $\Gamma$-graded quasi-Hom-Lie, $\Gamma$-graded Hom-Lie algebras, and graded quasi-Lie algebras of Witt type considered by Sigurdsson and Silvestrov in [11]. Recently, Ammar and Makhlouf [1] furthered this line of investigation by extending the main theorem from original paper by Hartwig, Larsson, and Silvestrov [7] to the $\mathbb{Z}_{2}$-graded case of Hom-Lie superalgebras. Also, Daniel Larsson has considered in [13] a modification of Hom-Lie algebras in connection to number theory and arithmetic geometry.

In the search of a counterpart of the associative algebras in the context of Hom-Lie algebras, Hom-associative algebras have been introduced by Makhlouf and Silvestrov in 2006 in [15], where it was shown that the commutator bracket of a Hom-associative algebra gives rise to a Hom-Lie algebra. Furthermore, in [15], a classification of Hom-Lie admissible algebras was established, Hom-Leibniz algebras, the subclass of quasi-Leibniz algebras, have been considered, notion of flexibility was extended to Hom-algebras, and flexible Hom-algebras have been characterized using Jordan and Lie parts of the multiplication. Also, in [15], a generalization of Hom-associative algebras, $G$-Hom-associative algebras, and their subclasses left symmetric Hom-algebras, Hom-pre-Lie algebras, or right symmetric Hom-algebras, and two other new classes of Hom-algebras have been introduced and $G$-Hom-associative algebras 
have been shown to be Hom-Lie admissible. The issue of constructing enveloping Homalgebra structures for Hom-Lie algebras has been considered by Yau in [19].

On the dual side, Hom-coalgebras, Hom-bialgabras, and Hom-Hopf algebras have been considered for the first time by Makhlouf and Silvestrov in [16] in 2007 and further studied by the same authors in [17] and also by Yau in [21]. Recently, Yau has continued this investigation in the direction of Hom-quantum groups in [22, 23]. Also, in 2009, following the works of Makhlouf, Silvestrov, and Yau, Hom-Hopf algebras were put in a general framework of monoidal categories by Caenepeel and Goyvaerts [3]. At the same time, on the side of algebra structures, formal deformations and elements of co-homology for Hom-associative and Hom-Lie algebras have been considered by Makhlouf and Silvestrov in [18], and elements of homology for Hom-algebras have been considered by Yau in [20]. Also, recently, Ataguema, Makhlouf, and Silvestrov introduced Hom-Nambu and Hom-Nambu-Lie algebras and $n$-ary versions of Hom-associative algebras in [2], and Makhlouf introduced Hom-Jordan algebras in [14].

Hom-associative algebras have been a subject of recent intensive study due to their rich structure theory and the fact that constructions coming from their classical counterparts have been found to transfer to a meaningful extent. Also helpful in this context is the availability of computational tools which greatly facilitate the search for examples and the proof of equational theorems about Hom-associative structures. Finally, inspiration has come from other types of Hom-algebras such as Hom-Lie algebras.

It was first pointed out by Fregier and Gohr in [4] that in the process of defining a twisted notion of, for instance, associativity or the Jacobi identity, there are some choices left on where to apply the twisting. At least in the Hom-associative category it does not seem to be the case that only one of the choices leads to an interesting theory. In [4], this leads to a systematic study of alternative types of Hom-associative algebras. In [3], an attempt is made to identify a single "correct" set of definitions for the Hom-associative and eventually the Hom-Hopf settings by approaching the problem of defining these notions from a categorytheoretical point of view.

In this article, we provide a common framework unifying all the types of Hom-associative algebras previously considered and many more exotic ones. We show that there is significant structure theory even in the context of such a strongly generalized notion of associativity. Finally, we discuss how conversely a related natural conjecture fails even under a mild generalization of the ordinary notion of Hom-associativity.

\section{Hom-associative algebras.}

Hom-associative algebras were introduced in [15], motivated by the need to obtain replacement of associative algebras in the context of Hom-Lie algebras providing also further ways of construction of Hom-Lie algebras. In [4], a study of alternative notions of Hom-associativity was started, essentially focusing on ways to use a single twisting map several times on both sides of the defining identity, while trying to preserve its symmetry and without considering cases where high powers of the twisting map appear. As a background, we quote the following table from [4] which summarizes the types considered there:

\begin{tabular}{|l|l|}
\hline$I_{1}$ & $\alpha(x) \star(y \star z)=(x \star y) \star \alpha(z)$ \\
\hline$I_{2}$ & $x \star(\alpha(y) \star z)=(x \star \alpha(y)) \star z$ \\
\hline$I_{3}$ & $x \star(y \star \alpha(z))=(\alpha(x) \star y) \star z$ \\
\hline
\end{tabular}




\begin{tabular}{|c|c|}
\hline$I I$ & $x \star \alpha(y \star z)=\alpha(x \star y) \star z$ \\
\hline$I I_{1}$ & $x \star(\alpha(y) \star \alpha(z))=(\alpha(x) \star \alpha(y)) \star z$ \\
\hline$I I_{2}$ & $\alpha(x) \star(y \star \alpha(z))=(\alpha(x) \star y) \star \alpha(z)$ \\
\hline$I I_{3}$ & $\alpha(x) \star(\alpha(y) \star z)=(x \star \alpha(y)) \star \alpha(z)$ \\
\hline
\end{tabular}

\begin{tabular}{|l|r|}
\hline$I I I$ & $\alpha(x \star(y \star z))=\alpha((x \star y) \star z)$ \\
\hline$I I I^{\prime}$ & $\alpha(x) \star \alpha(y \star z)=\alpha(x \star y) \star \alpha(z)$ \\
\hline$I I I^{\prime \prime}$ & $\alpha(x) \star(\alpha(y) \star \alpha(z))=(\alpha(x) \star \alpha(y)) \star \alpha(z)$ \\
\hline
\end{tabular}

In this paper, when we talk about a Hom-associative structure $(V, \star, \alpha)$ without specifying anything else, we mean that $(V, \star, \alpha)$ is Hom-associative in the usual sense, i.e., the binary operation satisfies the ordinary $\alpha$-twisted associativity relation $\alpha(x) \star(y \star z)=(x \star y) \star \alpha(z)$. Linearity of any of the maps is regularly implied only when a module structure is given on $V$.

For precision of terminology, we repeat from [4] the following general definition associated with the Hom-associativity types. Here $T$ denotes any of the types in the table quoted above.

Definition 2.1. A Hom-associative structure of type $T$ is a triple $(V, \star, \alpha)$ consisting of a set $V$ equipped with a binary operation $\star: V \times V \rightarrow V$ and a map $\alpha: V \rightarrow V$ satisfying the Hom-associativity condition corresponding to type $T$. Here, $T$ is understood to be one of the types in the table reproduced above.

Hom-rings and Hom-algebras are defined similarly, by imposing on $\alpha$ natural compatibility conditions, more specifically compatibility with the abelian group structure $(A,+)$ in the case of Hom-rings and linearity in the case of Hom-algebras.

As observed in [4], without additional constraints these types of Hom-associative structures seem to not be closely linked to each other, i.e., as far as we know a structure may be of any type without being of any other. But with additional constraints one gets a nontrivial theory. In particular, as a possible constraint the property of having a unit element was considered in [4]. Under this condition, a partial ordering of types was obtained with the traditional type $I_{1}$ ending up on top.

It should be noted however that in Hom-associative structures, several types of unitality make sense as was observed in $[3,4,5,6]$. Simultaneous investigation of the Hom-structures with various types of unitality is important and relations between the types in [4] are far less clear, e.g., in the case of weakly unital algebras.

In this paper we focus on the Hom-structures with the usual unitality condition, i.e., the existence of an element 1 in $V$ such that $1 \star x=x \star 1=x$ for all $x$ in $V$. In the rest of the paper unless stated otherwise, we assume all Hom-structures to be unital in this usual sense.

We will now recall the definition of Hom-monoids and the short discussion of their relation to Hom-algebras as they were given in [4]. Our main motivation is the use of Hom-monoids in the construction of the counterexample of Section 5 .

Definition 2.2. A Hom-monoid of type $T$ is a Hom-associative structure of type $T$ with unit.

There is a canonical way to associate to a Hom-associative algebra a Hom-monoid and to a Hom-monoid a Hom-associative algebra. We quote in this context the following example and remark from [4]. 
Example 2.3. Let $(V, \star,+, \alpha, 1)$ be a Hom-algebra of type $T$. Then the multiplicative structure $(V, \star, \alpha, 1)$ is a Hom-monoid also of type $T$.

Remark 2.4. Let $k$ be a commutative ring and let $(S, \tilde{\star}, \tilde{\alpha}, 1)$ be a Hom-monoid of type $T$. Let then $V$ be the free $k$-module over $S$ and define $\alpha: V \rightarrow V$ and $\star: V \times V \rightarrow V$ by linear extension of $\tilde{\alpha}: S \rightarrow S$, respectively, $\tilde{\star}: S \times S \rightarrow S$ to $V$. Then $(V, \star, \alpha, 1)$ is a unital Hom-associative algebra of type $T$. We denote the Hom-algebra so constructed from a Hom-monoid $S$ by $k[S]$.

\section{Sufficient conditions for associativity and hierarchy on types of unital Hom associative algebras}

One of the first natural problems about Hom-associative algebras is to determine how close they are to being associative. The simplest form of this problem is to determine conditions under which a Hom-associative algebra is itself associative. This problem has been considered in [5] for unital Hom-associative algebras in the usual sense. For some of the types considered in [4], there exist obvious associativity criteria, sometimes even in the absence of unitality constraints. But for many of the nonstandard types it is not obvious whether the known answers for the ordinary type generalize.

The aim of this section is to give an overview of the answers known to this question in the case of a Hom-associative algebra of type $I_{1}$ and recall the hierarchy among the different types, in order to motivate our subsequent investigations.

We recall first the following proposition providing sufficient conditions on the twisting map for a Hom-associative algebra to be associative [5].

Proposition 3.1. Let $(V, \star, \alpha, 1)$ be a unital Hom-associative structure of type $I_{1}$. Then the following hold.

(i) $(V, \star)$ is associative if $\alpha$ is surjective.

(ii) $(V, \star)$ is associative if $\alpha$ is injective.

(iii) If $\alpha$ is surjective, then $\alpha$ is also injective.

To finish our survey of results in this direction of associativity conditions for unital Homassociative structures, we remark that the condition of surjectivity can be relaxed for $V$ a Hom-algebra over a field by introducing conditions on the codimension of $\operatorname{Im}(\alpha)$ in $V$. For instance, $\operatorname{dim}(V / \operatorname{Im}(\alpha))=1$ is already sufficient to force associativity. For details, we refer the reader to [5].

The hierarchy for the relations among Hom-associative types $I$ and $I I$ can be summarized in the following proposition taken verbatim from [4].

Proposition 3.2. Let $(V, \star, 1, \alpha)$ be unital structure, then one has the following relations between the Hom-associativity types satisfied by $(V, \star, 1, \alpha)$ :

(a) $I I_{1} \Leftarrow I I \Leftrightarrow I_{1} \Rightarrow I_{3} \Rightarrow\left\{I_{2}, I I_{2}, I I_{3}\right.$ and $\left.I I_{1}\right\}$,

(b) $I_{2} \Rightarrow\left\{I I_{1} \Leftrightarrow I I_{3}\right\}$.

The point of recalling this hierarchy is that in a general theory for associativity conditions similar to the ones surveyed in the original type we will expect counterexamples to appear with greater likelihood in the lower levels of the hierarchy, since these are the least restricted. On the other hand, if we can find counterexamples to certain conjectures in the higher levels 
of the hierarchy, we know immediately that the conjecture in question is wrong for all types below. For instance, we will show in Section 5 that unital Hom-associative algebras of type $I_{3}$ are not necessarily associative if they have an injective twisting map. By this we will immediately know that the same counterexample works for any types which in terms of the hierarchy are more general.

The next section is devoted to the extension of Proposition 3.1 in a generalized framework which encompasses all of these types. But one has to be very careful in which sense this proposition is extended. For example, Proposition 3.1(ii) is false in the case of type $I_{3}$ as is shown by the counterexample given in the last section of this article.

\section{Generalized Hom-associative structures and automatic associativity conditions}

In this section, we introduce generalized Hom-associative structures that are built using several twisting maps and in particular include all types of Hom-associative structures discussed so far. For unital generalized Hom-structures we obtain in Theorem 4.2 sufficient conditions for associativity, generalizing Proposition 3.1.

Definition 4.1. A generalized Hom-associative structure is a tuple $\left(V, \star, \alpha_{1}, \alpha_{2}, \alpha_{3}, \alpha_{4}, \alpha_{5}\right)$ consisting of a set $V$, a binary operation $\star: V \times V \rightarrow V$ and five maps $\alpha_{j}: V \rightarrow V$, $j=1, \ldots, 5$, such that

$$
\alpha_{5}\left(\alpha_{1}(x) \star \alpha_{2}\left(\alpha_{3}(y) \star \alpha_{4}(z)\right)\right)=\alpha_{5}\left(\alpha_{2}\left(\alpha_{4}(x) \star \alpha_{3}(y)\right) \star \alpha_{1}(z)\right)
$$

Theorem 4.2. If a generalized Hom-structure $\left(V, \star, \alpha_{1}, \alpha_{2}, \alpha_{3}, \alpha_{4}, \alpha_{5}\right)$ has a unit element and if $\alpha_{1}, \alpha_{3}, \alpha_{4}$ are surjective and $\alpha_{2}, \alpha_{5}$ are injective, then $(V, \star)$ is associative.

The following corollary is one of the motivating applications of Theorem 4.2.

Corollary 4.3. If $(V, \star)$ is a nonassociative unital algebra, then the $\alpha$-twisted product

$$
x \circ y:=\alpha(x \star y)
$$

cannot be Hom-associative if the twisting map $\alpha$ is bijective.

Proof of Corollary 4.3. Hom-associativity for $\circ$ rewritten in terms of $\star$ is

$$
\alpha(\alpha(x) \star \alpha(y \star z))=\alpha(\alpha(x \star y) \star \alpha(z))
$$

which coincides with (4.1) when $\alpha_{3}=\alpha_{4}=\operatorname{id}_{V}$ and $\alpha_{1}=\alpha_{2}=\alpha_{5}=\alpha$. If $\alpha$ is bijective, then equivalently it is both surjective and injective, and hence $(V, \star)$ must be associative by Theorem 4.2 in contradiction with the assumption of nonassociativity.

In particular, this shows that the octonions do not admit a bijective twisting into a Homassociative algebra since they are unital and nonassociative.

The proof of Theorem 4.2 is more complicated and is based on several lemmas. First of all note that under the condition of injectivity of $\alpha_{5}$ in Theorem 4.2 the identity (4.1) can be rewritten equivalently without $\alpha_{5}$ as

$$
\alpha_{1}(x) \star \alpha_{2}\left(\alpha_{3}(y) \star \alpha_{4}(z)\right)=\alpha_{2}\left(\alpha_{4}(x) \star \alpha_{3}(y)\right) \star \alpha_{1}(z)
$$


In Theorem 4.2, the maps $\alpha_{1}, \alpha_{3}, \alpha_{4}$ are assumed to be surjective. In the sequel we choose some right-inverses $\beta_{1}, \beta_{3}, \beta_{4}$ of $\alpha_{1}, \alpha_{3}, \alpha_{4}$, respectively, $\left(\alpha_{j} \circ \beta_{j}=\mathrm{id}_{V}\right)$. Also, since $\alpha_{2}$ is assumed to be injective, we can choose some left-inverse $\beta_{2}$ of $\alpha_{2}$, that is, $\beta_{2} \circ \alpha_{2}=\mathrm{id} V$.

Using $\alpha_{3} \circ \beta_{3}=\mathrm{id}_{V}$, the map $\alpha_{3}$ can be removed from the generalized Hom-associativity axiom, which is thus equivalent to

$$
\alpha_{1}(x) \star \alpha_{2}\left(y \star \alpha_{4}(z)\right)=\alpha_{2}\left(\alpha_{4}(x) \star y\right) \star \alpha_{1}(z)
$$

The following direct consequence of generalized Hom-associativity (4.3) can be easily proved using $\alpha_{1} \circ \beta_{1}=\mathrm{id}_{V}$.

Lemma 4.4. Under conditions of Theorem 4.2, the following identity holds:

$$
\alpha_{1}(x) \star \alpha_{2}\left(y \star \alpha_{4}\left(\beta_{1}(z)\right)\right)=\alpha_{2}\left(\alpha_{4}(x) \star y\right) \star z
$$

By substitution of $\beta_{1}(x)$ in place of $x$, then using $\alpha_{1} \circ \beta_{1}=\mathrm{id}_{V}$ and finally setting $y=1$ and $z=1$ in (4.4), we get the following corollary.

\section{Corollary 4.5.}

$$
x \star \alpha_{2}\left(\alpha_{4}\left(\beta_{1}(1)\right)\right)=\alpha_{2}\left(\alpha_{4}\left(\beta_{1}(x)\right)\right)
$$

Lemma 4.6. Under conditions of Theorem 4.2, the following identity holds:

$$
\alpha_{4}\left(\beta_{1}\left(\alpha_{1}(x)\right)\right)=\alpha_{4}(x)
$$

Proof. By Corollary 4.5 and Lemma 4.4 with $y=z=1$, we have

$$
\begin{aligned}
& \alpha_{2}\left(\alpha_{4}\left(\beta_{1}\left(\alpha_{1}(x)\right)\right)\right) \stackrel{\text { Corollary }}{=}{ }^{4.5} \alpha_{1}(x) \star \alpha_{2}\left(\alpha_{4}\left(\beta_{1}(1)\right)\right) \\
& \stackrel{\text { Lemma }}{=}{ }^{4.4} \alpha_{2}\left(\alpha_{4}(x)\right)
\end{aligned}
$$

which is equivalent to (4.6) by the assumption of injectivity of $\alpha_{2}$.

Lemma 4.7. Under conditions of Theorem 4.2, the following identity holds:

$$
\alpha_{2}\left(\alpha_{4}\left(\beta_{1}(x)\right) \star y\right) \star z=x \star \alpha_{2}\left(y \star \alpha_{4}\left(\beta_{1}(z)\right)\right)
$$

Proof. The proof is obtained using (r.inv) the existence of a right inverse $\alpha_{1} \circ \beta_{1}=\operatorname{id}_{V}$ and generalized Hom-associativity as follows:

$$
\begin{aligned}
x \star \alpha_{2}\left(y \star \alpha_{4}\left(\beta_{1}(z)\right)\right) & \stackrel{\text { r.inv }}{=} \alpha_{1}\left(\beta_{1}(x)\right) \star \alpha_{2}\left(y \star \alpha_{4}\left(\beta_{1}(z)\right)\right) \\
& \stackrel{(4.3)}{=} \alpha_{2}\left(\alpha_{4}\left(\beta_{1}(x)\right) \star y\right) \star \alpha_{1}\left(\beta_{1}(z)\right) \\
& \stackrel{\text { r.inv }}{=} \alpha_{2}\left(\alpha_{4}\left(\beta_{1}(x)\right) \star y\right) \star z
\end{aligned}
$$

Substitution of $z=1$ into (4.7) leads to the following corollary.

Corollary 4.8. Under conditions of Theorem 4.2, the following identity holds:

$$
x \star \alpha_{2}\left(y \star \alpha_{4}\left(\beta_{1}(1)\right)\right)=\alpha_{2}\left(\alpha_{4}\left(\beta_{1}(x)\right) \star y\right)
$$


Putting $x=1$ in (4.8) and then changing in the obtained identity letter $y$ to letter $x$ leads to the following corollary.

Corollary 4.9. Under conditions of Theorem 4.2, the following identity holds:

$$
\alpha_{2}\left(\alpha_{4}\left(\beta_{1}(1)\right) \star x\right)=\alpha_{2}\left(x \star \alpha_{4}\left(\beta_{1}(1)\right)\right)
$$

The assumption that $\alpha_{2}$ is injective can be now applied to (4.9) to show that $\alpha_{4}\left(\beta_{1}(1)\right)$ is in the center of $(V, \star)$, that is, commutes with any other element in $V$.

Corollary 4.10. Under conditions of Theorem 4.2, the following identity holds:

$$
x \star \alpha_{4}\left(\beta_{1}(1)\right)=\alpha_{4}\left(\beta_{1}(1)\right) \star x
$$

Using (4.3) and $\alpha_{4} \circ \beta_{4}=\mathrm{id}_{V}$ we get also the following lemma.

Lemma 4.11. Under conditions of Theorem 4.2, the following identity holds:

$$
\alpha_{1}\left(\beta_{4}(x)\right) \star \alpha_{2}(y)=\alpha_{2}(x) \star \alpha_{1}\left(\beta_{4}(y)\right)
$$

Proof. The proof is obtained using (4.3) and $\alpha_{4} \circ \beta_{4}=\mathrm{id}_{V}$ :

$$
\begin{aligned}
\alpha_{1}\left(\beta_{4}(x)\right) \star \alpha_{2}(y) & \stackrel{\text { r.inv }}{=} \alpha_{1}\left(\beta_{4}(x)\right) \star \alpha_{2}\left(1 \star \alpha_{4}\left(\beta_{4}(y)\right)\right) \\
& \stackrel{(4.3)}{=} \alpha_{2}\left(\alpha_{4}\left(\beta_{4}(x)\right) \star 1\right) \star \alpha_{1}\left(\beta_{4}(y)\right) \\
& \stackrel{\text { r.inv }}{=} \alpha_{2}(x) \star \alpha_{1}\left(\beta_{4}(y)\right)
\end{aligned}
$$

Next lemma is obtained by combining (4.11), (4.9), (4.8), (4.6), and $\alpha_{4} \circ \beta_{4}=\mathrm{id}_{V}$.

Lemma 4.12. Under conditions of Theorem 4.2, the following identity holds:

$$
\alpha_{2}(x) \star \alpha_{1}\left(\beta_{4}\left(\alpha_{4}\left(\beta_{1}(1)\right) \star y\right)\right)=\alpha_{2}(x \star y)
$$

Proof. The proof is as follows:

$$
\begin{aligned}
\alpha_{2}(x) \star \alpha_{1}\left(\beta_{4}\left(\alpha_{4}\left(\beta_{1}(1)\right) \star y\right)\right) & \stackrel{(4.11)}{=} \alpha_{1}\left(\beta_{4}(x)\right) \star \alpha_{2}\left(\alpha_{4}\left(\beta_{1}(1)\right) \star y\right) \\
& \stackrel{(4.9)}{=} \alpha_{1}\left(\beta_{4}(x)\right) \star \alpha_{2}\left(y \star \alpha_{4}\left(\beta_{1}(1)\right)\right) \\
& \stackrel{(4.8)}{=} \alpha_{2}\left(\alpha_{4}\left(\beta_{1}\left(\alpha_{1}\left(\beta_{4}(x)\right)\right)\right) \star y\right) \\
& \stackrel{(4.6)}{=} \alpha_{2}\left(\alpha_{4}\left(\beta_{4}(x)\right) \star y\right) \\
& \stackrel{\text { r.inv }}{=} \alpha_{2}(x \star y)
\end{aligned}
$$

The next lemma follows using (4.11), the generalized Hom-associativity (4.3), and $\alpha_{4} \circ \beta_{4}=\operatorname{id}_{V}$.

Lemma 4.13. Under conditions of Theorem 4.2, the following identity holds:

$$
\alpha_{2}(x \star y) \star \alpha_{1}\left(\beta_{4}(z)\right)=\alpha_{2}(x) \star \alpha_{1}\left(\beta_{4}(y \star z)\right)
$$


Proof. First of all,

$$
\alpha_{2}(x) \star \alpha_{1}\left(\beta_{4}\left(y \star \alpha_{4}(z)\right)\right)=\alpha_{2}(x \star y) \star \alpha_{1}(z)
$$

which is proved as follows:

$$
\begin{aligned}
\alpha_{2}(x \star y) \alpha_{1}(z) & \stackrel{\text { r.inv }}{=} \alpha_{2}\left(\alpha_{4}\left(\beta_{4}(x)\right) \star y\right) \star \alpha_{1}(z) \\
& \stackrel{(4.3)}{=} \alpha_{1}\left(\beta_{4}(x)\right) \star \alpha_{2}\left(y \star \alpha_{4}(z)\right) \\
& \stackrel{(4.11)}{=} \alpha_{2}(x) \star \alpha_{1}\left(\beta_{4}\left(y \star \alpha_{4}(z)\right)\right)
\end{aligned}
$$

Substituting $\beta_{4}(z)$ in place of $z$ in (4.14) we get

$$
\begin{aligned}
\alpha_{2}(x \star y) \star \alpha_{1}\left(\beta_{4}(z)\right) & \stackrel{(4.14)}{=} \alpha_{2}(x) \star \alpha_{1}\left(\beta_{4}\left(y \star \alpha_{4}\left(\beta_{4}(z)\right)\right)\right) \\
& \stackrel{\text { r.inv }}{=} \alpha_{2}(x) \star \alpha_{1}\left(\beta_{4}(y \star z)\right)
\end{aligned}
$$

Lemma 4.14. Under conditions of Theorem 4.2, the following identity holds:

$$
\left(x \star \alpha_{4}\left(\beta_{1}(1)\right)\right) \star y=x \star\left(\alpha_{4}\left(\beta_{1}(1)\right) \star y\right)
$$

Proof. The identity

$$
\alpha_{2}\left(x \star \alpha_{4}\left(\beta_{1}(1)\right)\right) \star y=\alpha_{2}\left(x \star \alpha_{4}\left(\beta_{1}(y)\right)\right)
$$

can be proved as follows. Multiplying (4.9) on the right by $y$ and then using the identity obtained by substituting $(x, y, z)$ by $\left(\beta_{1}(1), x, y\right)$ in $(4.4)$, one gets

$$
\begin{aligned}
\alpha_{2}\left(x \star \alpha_{4}\left(\beta_{1}(1)\right)\right) \star y & =\alpha_{2}\left(\alpha_{4}\left(\beta_{1}(1)\right) \star x\right) \star y \\
& \stackrel{(4.4)}{=} \alpha_{1}\left(\beta_{1}(1)\right) \star \alpha_{2}\left(x \star \alpha_{4}\left(\beta_{1}(y)\right)\right) \\
& \stackrel{\text { r.inv }}{=} \alpha_{2}\left(x \star \alpha_{4}\left(\beta_{1}(y)\right)\right)
\end{aligned}
$$

The proof can now be completed along the following lines:

$$
\begin{aligned}
\alpha_{2}\left(\left(x \star \alpha_{4}\left(\beta_{1}(1)\right)\right) \star y\right) & \stackrel{(4.12)}{=} \alpha_{2}\left(x \star \alpha_{4}\left(\beta_{1}(1)\right)\right) \star \alpha_{1}\left(\beta_{4}\left(\alpha_{4}\left(\beta_{1}(1)\right) \star y\right)\right) \\
& \stackrel{(4.16)}{=} \alpha_{2}\left(x \star \alpha_{4}\left(\beta_{1}\left(\alpha_{1}\left(\beta_{4}\left(\alpha_{4}\left(\beta_{1}(1)\right) \star y\right)\right)\right)\right)\right) \\
& \stackrel{(4.6)}{=} \alpha_{2}\left(x \star \alpha_{4}\left(\beta_{4}\left(\alpha_{4}\left(\beta_{1}(1)\right) \star y\right)\right)\right) \\
& \stackrel{\text { r.inv }}{=} \alpha_{2}\left(x \star\left(\alpha_{4}\left(\beta_{1}(1)\right) \star y\right)\right)
\end{aligned}
$$

Finally, we are ready to proceed with the proof of the main Theorem 4.2.

Proof of Theorem 4.2. It suffices, by injectivity of $\alpha_{2}$, to prove that

$$
\alpha_{2}((x \star y) \star z)=\alpha_{2}(x \star(y \star z))
$$

Let us start by replacing $x$ by $x \star y$ in (4.12):

$$
\alpha_{2}((x \star y) \star z)=\alpha_{2}(x \star y) \star \alpha_{1}\left(\beta_{4}\left(\alpha_{4}\left(\beta_{1}(1)\right) \star z\right)\right)
$$


Thus, we get

$$
\begin{aligned}
\alpha_{2}((x \star y) \star z) & \stackrel{(4.14) \operatorname{and}(4.17)}{=} \alpha_{2}(x) \star \alpha_{1}\left(\beta_{4}\left(y \star \alpha_{4}\left(\beta_{4}\left(\alpha_{4}\left(\beta_{1}(1)\right) \star z\right)\right)\right)\right) \\
& \stackrel{(4.15) \operatorname{and}(4.10)}{=} \alpha_{2}(x) \star \alpha_{1}\left(\beta_{4}\left(\alpha_{4}\left(\beta_{1}(1)\right)\right) \star(y \star z)\right) \\
& \stackrel{(4.12)}{=} \alpha_{2}(x \star(y \star z))
\end{aligned}
$$

for the last equality we replaced $y$ by $y \star z$ in (4.12). By injectivity of $\alpha_{2}$ our result is equivalent to associativity.

\section{Counterexample}

We have seen that the observation that unital Hom-associative structures with surjective twisting map are associative has with Theorem 4.2 a counterpart in the generalized Homassociative setting. Looking at Theorem 4.2, it is natural to ask whether the surjectivity or injectivity assumptions on the $\alpha_{i}$ may be varied. Can we possibly reach the same conclusions, for example, by assuming that $\alpha_{4}$ is injective and the other twisting maps are as in the theorem?

In this section, we will answer this question in the negative. To understand why we take $\alpha_{4}$ instead of $\alpha_{1}$ for our first study of this kind of question, one should go back to the hierarchy of Hom-associative algebra types discussed in [4] and repeated in Section 2. Under the partial ordering of Hom-associativity conditions discussed there, in some sense the strongest condition apart from the original one was the type $I_{3}$ condition. By Theorem 4.2, we know immediately that a unital type $I_{3}$ Hom-associative structure is associative if the twisting map is surjective. We also know that in type $I_{1}$ the condition of surjectivity of $\alpha$ can be replaced by injectivity. This makes it natural to ask if some similar replacement is possible in type $I_{3}$. The rest of the section is devoted to the construction of a type $I_{3}$ Hom-monoid which serves as a counterexample.

Let $S$ be a set and let $\tilde{M}$ be the free magma with unit over $S$. Recall that $\tilde{M}$ can be realized by inductively defining in a first step a set $\tilde{S}$ consisting of the empty pair $e$, of pairs of elements in $S$, and finally of arbitrary pairs of elements of $\tilde{S}$ itself. In a second step, one introduces on this set an equivalence relationship generated by the relations $(x, e) \sim(e, x) \sim x$ for all $x \in \tilde{S}$. The set so obtained we call $\tilde{M}$. Taking as magma multiplication then the pairforming operation, or more precisely to the operation induced by pair-forming on the level of equivalence classes of elements of $\tilde{S}, \tilde{M}$ becomes the free magma with unit over $S$. Choosing then some $c \in S$, we can define a type $I_{3}$ Hom-monoid by identifying also pairs in $\tilde{M}$ which can be transformed into each other by a chain of relations of the form $(x, c) \sim(c, x)$ for any $x \in \tilde{M}$ and $(x,(y, z)) \sim((x, y), z)$ for $x, y, z \in \tilde{M}$ and $y$ a term containing $c$ in at least one place. It is clear that the partition into equivalence classes induced by these relations on $\tilde{M}$ is compatible with pairing as product. We will denote by $M$ the magma given by the set of equivalence classes on $\tilde{M}$ with the multiplication induced by pairing, i.e., $x \cdot y:=(x, y)$ for all $x, y \in M$. Define $\alpha: M \rightarrow M$ by $\alpha(x):=(c, x)$. Then $(M, \cdot, \alpha)$ is a Hom- $I_{3}$-monoid ${ }^{1}$.

Suppose now that $S$ is a set with at least two elements. Then we can choose an $s \in S$ such that $s \neq c$. None of the elementary transformations that we defined on $\tilde{M}$ to obtain $M$

\footnotetext{
${ }^{1}$ Note however that $M$ as defined is not the free $I_{3}$-associative monoid over $S$. For instance, the relation $x(((c x) x) x)=(x((c x) x)) x$, by definition valid in $M$ for all $x \in M$, is not generally valid in $I_{3}$-monoids and therefore not in general in the free $I_{3}$-monoid. Why we do not use the free $I_{3}$-monoid over $S$ should become apparent when we prove that $\alpha$ as defined is injective.
} 
can be applied to $(s,(s, s))$ and $((s, s), s)$, respectively, showing that these two elements are different in $M$ and that therefore $M$ is not associative.

If $M$ is to supply a counterexample to the conjecture that for a Hom-monoid of type $I_{3}$ injectivity of $\alpha$ implies associativity, we still need to prove that $\alpha$, i.e., multiplication by $c$, is indeed injective on $M$.

To do this, we will first introduce some notation. For $x \in M$ we say that $x$ is $c$-free if $c$ does not appear in $x$. It is clear that this is independent of choice of representative of $x$ in $\tilde{S}$. Also, we note that powers of $c$ are well defined and therefore write $c^{n}$ to denote an $n$-fold product of $c$ with itself irrespective of parenthesizing. Next, we realize that any $x \in M$ can be written in the form

$$
\left(\left(\ldots\left(\left(c^{n}, x_{1}\right), x_{2}\right) \ldots x_{m-1}\right), x_{m}\right)
$$

with $c$-free $x_{i}$. This is proven by induction on term structure. The base case, terms which are elements of $S$, is obvious. Now if we have $(x, y) \in \tilde{M}$, we can by assumption find representations of $x$ and $y$ in the form

$$
x \sim\left(\left(\ldots\left(\left(c^{n}, x_{1}\right), x_{2}\right), \ldots x_{m-1}\right), x_{m}\right)
$$

and

$$
y \sim\left(\left(\ldots\left(\left(c^{r}, y_{1}\right), y_{2}\right), \ldots y_{s-1}\right), y_{s}\right)
$$

with $c$-free $x_{i}$ and $y_{i}$. Now if $r=0$, we see that $(x, y)$ itself is already in the claimed form. If on the other hand $r \neq 0$, we can use the fact that we have $(u v) w=u(v w)$ whenever $v$ is a term containing $c^{r}$ to see that

$$
\left.(x, y) \sim\left(\left(\left(\left(\left(\ldots\left(\left(c^{n}, x_{1}\right), x_{2}\right) \ldots x_{m-1}\right), x_{m}\right),\left(c^{r}, y_{1}\right)\right), y_{2}\right), \ldots y_{s-1}\right), y_{s}\right)
$$

Here, $c^{r}$ can still be drawn into the part of term corresponding to $x$. Since $c^{r}$ commutes with the $x_{i}$, we can iterate this process until we reach the form

$$
\left.(x, y) \sim\left(\left(\left(\left(\ldots\left(c^{n+r}, x_{1}\right), x_{2}\right), \ldots x_{m-1}\right), x_{m}\right), y_{1}\right), \ldots y_{s}\right)
$$

We see now that this "normal form" is completely characterized by the exponent of $c$ inside and the sequence $x_{1}, \ldots x_{m}, y_{1}, \ldots, y_{s}$ of $c$-free terms appearing afterwards. Noting that none of the admissible term rewriting rules allow one to change $c$-free subterms of a term, the order inside a term of $c$-free subterms, or the number of occurrences of $c$ in the whole term, we conclude that this normal form is uniquely defined for each $x \in M$. But, the only effect of multiplication by $c$ on the normal form of an element of $M$ is to raise the inner exponent by one. This is clearly a reversible operation, hence proving injectivity of multiplication by $c$.

\section{Acknowledgements}

This work was supported from the Swedish part by the Swedish Research Council, the Swedish Foundation for International Cooperation in Research and Higher Education (STINT), the Crafoord Foundation, the Royal Physiographic Society in Lund, and the Royal Swedish Academy of Sciences. We want to gratefully acknowledge the support of the research grant R1F105L15 of the University of Luxembourg (Martin Schlichenmaier).

We also acknowledge the assistance from the first-order logic automatic deduction software Prover9 used by us when discovering the proofs in Section 4 . 


\section{References}

[1] F. Ammar and A. Makhlouf. Hom-Lie superalgebras and Hom-Lie admissible superalgebras. Preprint arXiv:0906.1668v2, 2009.

[2] H. Ataguema, A. Makhlouf, and S. Silvestrov. Generalization of $n$-ary Nambu algebras and beyond. J. Math. Phys., 50 (2009), 083501.

[3] S. Caenepeel and I. Goyvaerts. Hom-Hopf algebras. Preprint arXiv:0907.0187, 2009.

[4] Y. Frégier and A. Gohr. On Hom type algebras. Preprint arXiv:0903.3393, 2009.

[5] Y. Frégier and A. Gohr. On unitality conditions for Hom-associative algebras. Preprint arXiv:0904.4874, 2009.

[6] A. Gohr. On Hom-algebras with surjective twisting. Preprint arXiv:0906.3270, 2009.

[7] J. T. Hartwig, D. Larsson, and S. D. Silvestrov. Deformations of Lie algebras using $\sigma$-derivations. J. Algebra, 295 (2006), 314-361.

[8] D. Larsson and S. D. Silvestrov. Quasi-Hom-Lie algebras, central extensions and 2-cocycle-like identities. J. Algebra, 288 (2005), 321-344.

[9] D. Larsson and S. D. Silvestrov. Quasi-Lie algebras. In "Noncommutative Geometry and Representation Theory in Mathematical Physics". J. Fuchs, J. Mickelsson, G. Rosenblioum, A. Stolin, and A. Westerberg, Eds. Contemp. Math., 391, American Mathematical Society, Providence, RI, 2005, 241-248.

[10] D. Larsson and S. D. Silvestrov. Graded quasi-Lie agebras. Czechoslovak J. Phys., 55 (2005), 1473-1478.

[11] G. Sigurdsson, and S. D. Silvestrov. Graded quasi-Lie algebras of Witt type. Czechoslovak J. Phys., 56 (2006), 1287-1291.

[12] D. Larsson and S. D. Silvestrov. Quasi-deformations of $\mathfrak{s l}_{2}(\mathbb{F})$ using twisted derivations. Comm. Algebra, 35 (2007), 4303-4318.

[13] D. Larsson. Arithmetic Witt-Hom-Lie algebras. J. Gen. Lie Theory Appl., 3 (2009).

[14] A. Makhlouf. Hom-alternative algebras and Hom-Jordan algebras. Preprint arXiv:0909.0326, 2009.

[15] A. Makhlouf and S. D. Silvestrov. Hom-algebra structures. J. Gen. Lie Theory Appl., 2 (2008), 51-64.

[16] A. Makhlouf and S. D. Silvestrov. Hom-Lie admissible Hom-coalgebras and Hom-Hopf algebras. In "Generalized Lie Theory in Mathematics, Physics and Beyond". S. Silvestrov, E. Paal, V. Abramov, and A. Stolin, Eds. Springer-Verlag, Berlin, 2009, 189-206. Preprints in Mathematical Sciences, Lund University, Centre for Mathematical Sciences, Centrum Scientiarum Mathematicarum (2007:25) LUTFMA-5091-2007 and in arXiv:0709.2413 [math.RA] (2007).

[17] A. Makhlouf and S. D. Silvestrov. Hom-Algebras and Hom-Coalgebras. J. Algebra Appl. (to be published). Preprints in Mathematical Sciences, Lund University, Centre for Mathematical Sciences, Centrum Scientiarum Mathematicarum (2008:19) LUTFMA-5103-2008 and in arXiv:0811.0400 [math.RA] (2008).

[18] A. Makhlouf and S. D. Silvestrov. Notes on Formal deformations of Hom-Associative and HomLie algebras. Forum Math. (to be published).

[19] D. Yau. Enveloping algebra of Hom-Lie algebras. J. Gen. Lie Theory Appl., 2 (2008), 95-108.

[20] D. Yau. Hom-algebras and Homology. J. Lie Theory, 19 (2009), 409-421.

[21] D. Yau. Hom-bialgebras and comodule algebras. Preprint arXiv:0810.4866, 2008.

[22] D. Yau. Hom-quantum groups I: quasi-triangular Hom-bialgebras. Preprint arXiv:0906.4128, 2009.

[23] D. Yau. Hom-quantum groups II: cobraided Hom-bialgebras and Hom-quantum geometry. Preprint arXiv:0907.1880, 2009. 